\title{
Ranking DMUs by Calculating the Interval Efficiency with a Common Set of Weights in DEA
}

\author{
Vahideh Rezaie, ${ }^{1}$ Tahir Ahmad, ${ }^{1}$ Siti-Rahmah Awang, \\ Masumeh Khanmohammadi, ${ }^{3}$ and Normah Maan ${ }^{1}$ \\ ${ }^{1}$ Department of Mathematical Sciences, Faculty of Science and Ibnu Sina Institute, Nanotechnology Research Alliance, \\ Universiti Teknologi Malaysia, Skudai, 81310 Johor Bahru, Malaysia \\ ${ }^{2}$ Faculty of Management, Universiti Teknologi Malaysia, Skudai, 81310 Johor Bahru, Malaysia \\ ${ }^{3}$ Department of Mathematics, Faculty of Science, Islamic Azad University, Islamshahr Branch, Tehran 11369, Iran
}

Correspondence should be addressed to Vahideh Rezaie; vahidehrezaie@yahoo.com and Tahir Ahmad; tahir@ibnusina.utm.my

Received 21 November 2013; Revised 22 April 2014; Accepted 5 May 2014; Published 1 June 2014

Academic Editor: Ch. Tsitouras

Copyright (C) 2014 Vahideh Rezaie et al. This is an open access article distributed under the Creative Commons Attribution License, which permits unrestricted use, distribution, and reproduction in any medium, provided the original work is properly cited.

\begin{abstract}
To evaluate the performance of decision making units (DMUs), data envelopment analysis (DEA) was introduced. Basically, the traditional DEA scheme calculates the best relative efficiency score (i.e., the "optimistic" efficiency) of each DMU with the most favorable weights. A decision maker may be unable to compare and fully rank the efficiencies of different DMUs that are calculated using these potentially distinct sets of weights on the same basis. Based on the literature, the assignable worst relative efficiency score (i.e., the "pessimistic" efficiency) for each DMU can also be determined. In this paper, the best and the worst relative efficiencies are considered simultaneously. To measure the overall performance of the DMUs, an integration of both the best and the worst relative efficiencies is considered in the form of an interval. The advantage of this efficiency interval is that it provides all of the possible efficiency values and an expanded overview to the decision maker. The proposed method determines the lower-and upper-bounds of the interval efficiency over a common set of weights. To demonstrate the implementation of the introduced method, a numerical example is provided.
\end{abstract}

\section{Introduction}

The traditional data envelopment analysis (DEA) [1] was established based on the efficient frontier concept in which the best efficiency score assignable to each DMU is determined. Charnes et al. [1] introduced a DEA method in which the most favorable weights for each DMU are discovered by considering the best efficiency score for the DMU. These efficiency scores are used as a basis to compare the performance of all of the DMUs.

The performances of DMUs from the "optimistic" and "pessimistic" efficiency viewpoints are evaluated by Entani et al. [2]. They used the "optimistic" and "pessimistic" efficiencies to create an interval. Based on their studies [2], the efficiency of a DMU is given in the form of an interval and is between the "optimistic" and "pessimistic" values. However, the method proposed by Entani et al. [2] has a critical defect in which some of the input and output data are not considered. Wang and Luo [3] introduced a DEA model in which the terms "ideal DMU" (IDMU) and "anti-ideal DMU" (ADMU) are used as two virtual DMUs to compute the "optimistic" and "pessimistic" efficiencies, respectively. Wang and Yang [4] introduced a pair of bounded DEA methods to evaluate the overall performance of each DMU. The "optimistic" and "pessimistic" efficiencies for each DMU are measured by utilizing the input and output information to the greatest extent possible. To assess the overall performance of each DMU, both "optimistic" and "pessimistic" efficiencies must be considered simultaneously [5]. In [6], Azizi introduced new DEA schemes to conduct a DEA analysis based on the concepts of "optimistic" and "pessimistic" efficiencies. These schemes, which are used to measure the interval efficiencies 
of DMUs, have upper- and lower-bounds. Azizi used the Aindex term developed by Sengupta and Pal [7] to compare and rank the efficiency intervals of DMUs.

Basically, the traditional (Charnes, Cooper, and Rhodes) CCR DEA scheme calculates the efficiency of each DMU by utilizing only the most favorable weights for DMU. The decision maker may be unable to compare and rank the efficiencies of different DMUs that were calculated using various sets of weights on the same basis [8]. However, more than one DMU can be evaluated as an efficient DMU due to the inherent flexibility in the weights' selection. Consequently, a decision maker may be unable to fully discriminate between all of the DMUs [9]. Some solutions to overcome these problems are reviewed in [9]. One of them is the common weights DEA method. In [10], a DEA model is proposed to determine the common weights based on the maximization of the sum of the DMUs' efficiencies. In [11, 12], to calculate a set of common weights, the average of various sets of weights is used. The average is achieved by performing an unbounded DEA model. They also suggested maximizing the average DMUs' efficiency and maximizing the number of efficient units as other methods to obtain the common set of weights. Sinuany-Stern and Friedman [13] proposed a nonlinear discriminated analysis to obtain a common set of weights. In Jahanshahloo et al. [14], two methods are introduced to obtain the set of common weights to rank the efficient DMUs using comparisons with ideal and special lines. Wang et al. [8] proposed a method based on regression analysis in which the common weights are determined from the efficiency-fitting viewpoint. The traditional DEA efficiencies are considered as the target efficiencies. Andersen and Petersen [15] evaluated the efficiency of a DMU by assuming that it may exceed the conventional score of 1.0. They made a comparison of the DMU under evaluation by means of a linear composition of other DMUs, whereby the observations of the DMU under evaluation are excluded. Cook et al. developed prioritization method [16]. It ranks the efficient DMUs. In their method, the DMUs with equal scores on the boundary are divided by imposing a limitation on the weights in a DEA analysis. Jahanshahloo et al. [17] proposed an $l_{1}$-norm method that addresses some of the shortcomings of the Andersen and Petersen (AP) method.

In this paper, both the best and the worst relative efficiencies are applied simultaneously. To measure the overall performance of the DMUs, an integration of both "optimistic" and "pessimistic" efficiencies is considered in the form of an interval. The proposed method determines the lower- and upper-bounds of the interval efficiency with a common set of weights. Then, the DMUs are ranked using this interval. Hence, all of the DMUs have a common set of weights and are compared simultaneously.

The rest of this paper is organized as follows: in Section 2, the DEA methods for calculating the "optimistic" and "pessimistic" efficiencies are introduced, related works are presented in Section 3, and the proposed method is introduced in Section 4. The results and discussion are given in Section 5. Finally, the paper is concluded in Section 6.

\section{Evaluation of the "Optimistic" and "Pessimistic" Efficiencies Using DEA Models}

2.1. The CCR Model for Evaluating the "Optimistic" Efficiencies of DMUs. Suppose that there are $n$ DMUs under evaluation such that each of them has $m$ inputs and $s$ outputs. The input and output values of $\mathrm{DMU}_{j}$ are defined as $x_{i j}(i=1, \ldots, m)$ and $y_{r j}(r=1, \ldots, s)$, respectively, for $j=1, \ldots, n$, and all of these values are known and positive. Based on the definition of efficiency, $\mathrm{DMU}_{j}$ 's efficiency is defined as follows:

$$
\theta_{j}=\frac{\sum_{r=1}^{s} u_{r} y_{r j}}{\sum_{i=1}^{m} v_{i} x_{i j}}, \quad j=1, \ldots, n,
$$

where $u_{r}(r=1, \ldots, s)$ and $v_{i}(i=1, \ldots, m)$ are the weights for the $r$ th output and the $i$ th input, respectively. Charnes et al. [1] introduced a known CCR model to differentiate the efficiency of $\mathrm{DMU}_{j}$ from the other DMUs. In CCR model, the best relative efficiency of the DMUs is measured. This efficiency is calculated as the following linear programming scheme:

$$
\begin{array}{ll}
\max & \theta_{o}=\sum_{r=1}^{s} u_{r} y_{r o} \\
\text { s.t. } & \sum_{r=1}^{s} u_{r} y_{r j}-\sum_{i=1}^{m} v_{i} x_{i j} \leq 0, \quad j=1, \ldots, n, \\
& \sum_{i=1}^{m} v_{i} x_{i o}=1, \\
& u_{r}, v_{i} \geq \varepsilon, \quad r=1, \ldots, s ; i=1, \ldots, m .
\end{array}
$$

In model (2), the subscript " $o$ " represents the DMU under evaluation; $v_{i}(i=1, \ldots, m)$ and $u_{r}(r=1, \ldots, s)$ are the variables for decision making. As $\varepsilon$ is a non-Archimedean infinitesimal, all weights are positive and it is impossible to have zero weights because of imposing $\varepsilon$ in DEA models. Amin and Toloo [18] presented an algorithm for computing the appropriate non-Archimedean $\varepsilon$ in DEA models. $\mathrm{DMU}_{o}$ is said to be "DEA efficient" or "optimistic efficient" when there is a set of positive weights $u_{r}^{*}(r=1, \ldots, s)$ and $v_{i}^{*}(i=1, \ldots, m)$ that make $\theta^{*}=1$; otherwise, DMU is said to be "DEA nonefficient" or "optimistic nonefficient." In conjunction, the "DEA efficient" DMUs define an efficient frontier. Throughout the paper, the superscript “*” is used to display the optimum amount of the variables.

2.2. The DEA Model for Evaluating the "Pessimistic" Efficiencies of DMUs. In the CCR model [1], the "optimistic" efficiency of DMUs is measured by maximizing it in the range of nonnegative real numbers less than or equal to one. The efficiency of a DMU is said to be the "pessimistic" efficiency or the worst relative efficiency when the measured efficiency of the DMU is greater than or equal to one. Using the below 
TABLE 1: Data for 10 DMUs with one input and two outputs.

\begin{tabular}{lccc}
\hline DMU & Input & Output 1 & Output 2 \\
\hline A & 1 & 1 & 8 \\
B & 1 & 2 & 3 \\
C & 1 & 2 & 6 \\
D & 1 & 3 & 3 \\
E & 1 & 3 & 7 \\
F & 1 & 4 & 2 \\
G & 1 & 4 & 5 \\
H & 1 & 5 & 2 \\
I & 1 & 6 & 2 \\
J & 1 & 7 & 1 \\
\hline
\end{tabular}

"pessimistic" DEA scheme [19], the "pessimistic" efficiency of $\mathrm{DMU}_{o}$ can be measured as follows:

$$
\begin{array}{ll}
\min & \varphi_{o}=\sum_{r=1}^{s} u_{r} y_{r o} \\
\text { s.t. } & \sum_{r=1}^{s} u_{r} y_{r j}-\sum_{i=1}^{m} v_{i} x_{i j} \geq 0, \quad j=1, \ldots, n, \\
& \sum_{i=1}^{m} v_{i} x_{i o}=1, \\
& u_{r}, v_{i} \geq \varepsilon, \quad r=1, \ldots, s ; i=1, \ldots, m .
\end{array}
$$

$\mathrm{DMU}_{o}$ is called the DEA inefficient or "pessimistic inefficient" DMU when there is a set of positive weights $u_{r}^{*}(r=1, \ldots, s)$ and $v_{i}^{*}(i=1, \ldots, m)$ that makes $\varphi^{*}=1$; otherwise, it is called "DEA non-inefficient" or "pessimistic non-inefficient." It is obvious that "pessimistic non-inefficient" is not synonymous with "optimistic efficient." An inefficient frontier is defined by all of the "pessimistic inefficient" DMUs.

In contrast to the introduced CCR model (2), which is called "optimistic" DEA models, the "pessimistic" DEA scheme introduced in (3) seeks the most unfavorable weights for DMUs. By using two-dimensional output and onedimensional input data from Entani et al. [2], as in Table 1, the differences between "DEA efficient," "DEA nonefficient," "DEA inefficient," and "DEA non-inefficient" DMUs are demonstrated. For the sake of convenience, all of the inputs are normalized. In addition, the "optimistic" and "pessimistic" efficiency scores of 10 DMUs are obtained based on DEA models (2) and (3), which are shown in Table 2. Figure 1 illustrates the efficient and inefficient frontiers of this dataset. $\mathrm{DMU}_{\mathrm{A}}, \mathrm{DMU}_{\mathrm{E}}$, and $\mathrm{DMU}_{\mathrm{J}}$, which are located on the efficient frontier, are called "DEA efficient" or "optimistic efficient" DMUs. The other DMUs are termed "DEA nonefficient" in relation to the efficient frontier. Moreover, $\mathrm{DMU}_{\mathrm{A}}, \mathrm{DMU}_{\mathrm{B}}, \mathrm{DMU}_{\mathrm{F}}$, and $\mathrm{DMU}_{\mathrm{J}}$, which are located on the inefficient frontier, are specified as "DEA inefficient" or "pessimistic inefficient." Other DMUs are termed as "DEA non-inefficient" in relation to the inefficient frontier. Some DMUs, which belong to the set of "DEA efficient" and
"DEA inefficient" DMUs $\left(\mathrm{DMU}_{\mathrm{A}}\right.$ and $\left.\mathrm{DMU}_{\mathrm{J}}\right)$, are located on both the efficient and inefficient frontiers. However, the exact positions of these DMUs $\left(\mathrm{DMU}_{\mathrm{A}}\right.$ and $\left.\mathrm{DMU}_{\mathrm{J}}\right)$ are not specified regardless of whether they are situated on the efficient or the inefficient frontiers. Entani et al. [2] proposed the concept of interval efficiency to meet this drawback. The concept will be explained in the next section.

\section{Related Works}

3.1. A Review of Entani et al.s DEA Model. Entani et al.s [2] DEA models are reviewed in which an efficiency interval for each DMU, along with upper- and lower-bounds mathematical programming schemes, is introduced as follows:

$$
\begin{aligned}
\max / \min & \theta_{o}=\frac{\sum_{r=1}^{s} u_{r} y_{r o} / \sum_{i=1}^{m} v_{i} x_{i o}}{\max _{j}\left\{\sum_{r=1}^{s} u_{r} y_{r j} / \sum_{i=1}^{m} v_{i} x_{i j}\right\}} \\
\text { s.t. } & u_{r}, v_{i} \geq \varepsilon, \quad r=1, \ldots, s ; i=1, \ldots, m .
\end{aligned}
$$

The upper-bound model is changed to scheme (5), and this model has an optimal value that can be solved using model (2):

$$
\begin{aligned}
& \max \quad \theta_{o}^{U}=\frac{\sum_{r=1}^{s} u_{r} y_{r o}}{\sum_{i=1}^{m} v_{i} x_{i o}} \\
& \text { s.t. } \max _{j}\left\{\frac{\sum_{r=1}^{s} u_{r} y_{r j}}{\sum_{i=1}^{m} v_{i} x_{i j}}\right\}=1, \quad j=1, \ldots, n \text {, } \\
& u_{r}, v_{i} \geq \varepsilon, \quad r=1, \ldots, s ; i=1, \ldots, m, \\
& \Longrightarrow \max \quad \theta_{o}^{U}=\frac{\sum_{r=1}^{s} u_{r} y_{r o}}{\sum_{i=1}^{m} v_{i} x_{i o}} \\
& \text { s.t. } \quad \frac{\sum_{r=1}^{s} u_{r} y_{r j}}{\sum_{i=1}^{m} v_{i} x_{i j}} \leq 1, \quad j=1, \ldots, n, \\
& u_{r}, v_{i} \geq \varepsilon, \quad r=1, \ldots, s ; i=1, \ldots, m .
\end{aligned}
$$

Moreover, the lower-bound is converted to (6) as follows:

$$
\begin{array}{ll}
\min & \theta_{o}^{L}=\frac{\sum_{r=1}^{s} u_{r} y_{r o}}{\sum_{i=1}^{m} v_{i} x_{i o}} \\
\text { s.t. } & \max _{j}\left\{\frac{\sum_{r=1}^{s} u_{r} y_{r j}}{\sum_{i=1}^{m} v_{i} x_{i j}}\right\}=1, \\
& u_{r}, v_{i} \geq \varepsilon, \quad r=1, \ldots, s ; i=1, \ldots, m .
\end{array}
$$

Model (6) cannot be substituted with an equivalent LP scheme. Let $\sum_{r=1}^{s} u_{r} y_{r j} / \sum_{i=1}^{m} v_{i} x_{i j}=1$ for any "DEA efficient" DMU; then (6) is separated into $p$ suboptimization problems $\left(j=J_{1}, \ldots, J_{p}\right)$ in which $p$ is the number of "DEA efficient" 
TABLE 2: The interval efficiencies of Entani et al's model [2] and Azizi's model [6] and the relative efficiencies for 10 DMUs.

\begin{tabular}{lccccc}
\hline DMU & $\begin{array}{c}\text { Optimistic efficiency } \\
\text { (model 2) }\end{array}$ & $\begin{array}{c}\text { Pessimistic } \\
\text { efficiency (model 3) }\end{array}$ & $\begin{array}{c}\text { Interval efficiency } \\
\text { (Entani et al.s model [2]) }\end{array}$ & $\begin{array}{c}\text { Interval efficiency } \\
\text { (Azizi's model [6]) }\end{array}$ & $\begin{array}{c}\text { Rank } \\
(\text { Azizi's method [6]) }\end{array}$ \\
\hline A & 1.0000 & 1.0000 & {$[0.1428,1.0000]$} & {$[0.1814,1.0000]$} & 3 \\
B & 0.5217 & 1.0000 & {$[0.2857,0.5217]$} & {$[0.1814,0.5217]$} & 10 \\
C & 0.8235 & 1.2308 & {$[0.2857,0.8235]$} & {$[0.2233,0.8235]$} & 9 \\
D & 0.6522 & 1.1250 & {$[0.3750,0.6522]$} & {$[0.2041,0.6522]$} & 1 \\
E & 1.0000 & 1.6923 & {$[0.4285,1.0000]$} & {$[0.3070,1.0000]$} & 8 \\
F & 0.6957 & 1.0000 & {$[0.2500,0.6957]$} & {$[0.1814,0.6957]$} & 2 \\
G & 0.9565 & 1.7500 & {$[0.5714,0.9565]$} & {$[0.3175,0.9565]$} & 7 \\
H & 0.8261 & 1.1000 & {$[0.2500,0.8261]$} & {$[0.1995,0.8261]$} & 5 \\
I & 0.9565 & 1.2000 & {$[0.2500,0.9565]$} & {$[0.2177,0.9565]$} & 3 \\
J & 1.0000 & 1.0000 & {$[0.1250,1.0000]$} & {$[0.1814,1.0000]$} & \\
\hline
\end{tabular}

units and $J_{1}, \ldots, J_{p}$ are the "DEA efficient" DMUs. Model (6) can be simplified by converting it into the $p$ LP as follows:

$$
\begin{array}{ll}
\min & \theta_{o j}^{L}=\sum_{r=1}^{s} u_{r} y_{r o} \\
\text { s.t. } & \sum_{r=1}^{s} u_{r} y_{r j}-\sum_{i=1}^{m} v_{i} x_{i j}=0, \\
& \sum_{i=1}^{m} v_{i} x_{i o}=1, \\
& u_{r}, v_{i} \geq \varepsilon, \quad r=1, \ldots, s ; i=1, \ldots, m .
\end{array}
$$

Suppose that $\theta_{o j}^{L *}$ is the value of the optimum objective function for the proposed LP model in (7); it is clear that, for $j=o$, the value of $\theta_{o j}^{L *}$ equals 1 . The lower-bound efficiency of $\mathrm{DMU}_{o}$ can be calculated as follows:

$$
\theta_{o}^{L *}=1 \wedge \min _{j \neq o}\left\{\theta_{o j}^{L *}\right\} \quad \text { in which } a \wedge b=\min \{a, b\} .
$$

Finally, the interval efficiency of $\mathrm{DMU}_{o}$ is given as $\left[\theta_{o}^{L *}, \theta_{o}^{U *}\right]$, with $\theta_{o}^{U *}$ which is the value of the optimum objective function for the upper-bound introduced in (5).

Azizi [6] analyzed Entani et al.'s method. According to Azizi's assessment [6], model (7) has only two constraints. Thus, without considering the numbers of inputs and outputs in the problem under evaluation, only two variables can be nonzero: one of them is an input for one weight and the other variable is an output for the other weight. Therefore, Entani et al.'s model [2] calculates the "pessimistic" efficiency for different DMUs by considering only one input and one output. Moreover, the model proposed by (7) cannot identify the exact inefficient DMUs or the inefficient frontier.

Azizi [6] showed the drawback of Entani et al's method that some weights, which are obtained from the lowerbound efficiency model in (7), give an efficiency score greater than one for some DMUs. Consequently, the assumption $\max _{j}\left\{\sum_{r=1}^{s} u_{r} y_{r j} / \sum_{i=1}^{m} v_{i} x_{i j}\right\}=1$, which is a constraint for lower-bound model (6), is clearly incompatible with these results.

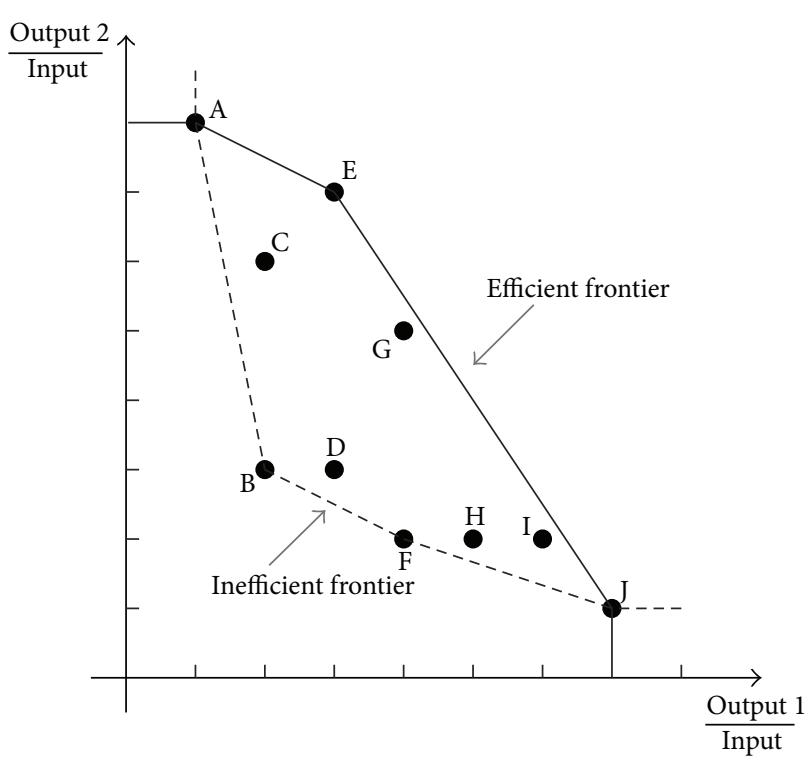

FIGURE 1: Efficient and inefficient frontiers for 10 DMUs.

3.2. A Review of Azizi's DEA Model. Azizi [6] adjusted the "pessimistic" efficiency as follows.

By considering $0<\alpha \leq 1$ as the adjustment coefficient, the adjusted "pessimistic" efficiencies are illustrated by $\widetilde{\varphi}_{j}^{*}=$ $\alpha \varphi_{j}^{*}(j=1, \ldots, n)$ and satisfy the condition $\widetilde{\varphi}_{j}^{*}=\alpha \varphi_{j}^{*} \leq$ $\theta_{j}^{*}(j=1, \ldots, n)$. That is, $\alpha \leq \min _{j=1, \ldots, n}\left\{\theta_{j}^{*} / \varphi_{j}^{*}\right\}$. Thus, the efficiency interval of $\mathrm{DMU}_{j}$ is given by $\left[\alpha \varphi_{j}^{*}, \theta_{j}^{*}\right](j=$ $1, \ldots, n)$. The IDMU was defined by Azizi as a virtual DMU for which the input and output are as follows:

$$
\begin{array}{ll}
x_{i}^{\min }=\min _{j}\left\{x_{i j}\right\}, & i=1, \ldots, m, \\
y_{r}^{\max }=\max _{j}\left\{y_{r j}\right\}, & r=1, \ldots, s .
\end{array}
$$


Based on the definition of efficiency, the efficiency of IDMU is defined as follows:

$$
\varphi_{\mathrm{IDMU}}=\frac{\sum_{r=1}^{s} u_{r} y_{r}^{\max }}{\sum_{i=1}^{m} v_{i} x_{i}^{\min }} .
$$

Suppose $\varphi_{\text {IDMU }}^{*}$ is the "pessimistic" efficiency of IDMU. Then, by considering model (3), the linear programming model becomes

$$
\begin{array}{ll}
\min & \varphi_{\mathrm{IDMU}}=\sum_{r=1}^{s} u_{r} y_{r}^{\max } \\
\text { s.t. } & \sum_{r=1}^{s} u_{r} y_{r j}-\sum_{i=1}^{m} v_{i} x_{i j} \geq 0, \quad j=1, \ldots, n, \\
& \sum_{i=1}^{m} v_{i} x_{i}^{\min }=1, \\
& u_{r}, v_{i} \geq \varepsilon, \quad r=1, \ldots, s ; i=1, \ldots, m .
\end{array}
$$

The parameter $\alpha$ is defined with the condition $\alpha \varphi_{j}^{*} \leq$ $\theta_{j}^{*}(j=1, \ldots, n)$ for all $\left[\alpha \varphi_{j}^{*}, \theta_{j}^{*}\right]$ with $j=1, \ldots, n$,

$$
\min _{j=1, \ldots, n}\left\{\frac{\theta_{j}^{*}}{\varphi_{j}^{*}}\right\} \geq \frac{\min _{j=1, \ldots, n}\left\{\theta_{j}^{*}\right\}}{\max _{j=1, \ldots, n}\left\{\varphi_{j}^{*}\right\}} \geq \frac{\theta_{\text {min }}^{*}}{\varphi_{\text {IDMU }}^{*}} .
$$

The parameter $\alpha$ is trivial when $\alpha=\theta_{\min }^{*} / \varphi_{\text {IDMU }}^{*}$. Therefore, the DMUs' efficiencies are measured in the interval $[\alpha, 1]$. This idea is reflected by the next two fractional programming schemes:

$$
\begin{aligned}
\max / \min & \frac{\sum_{r=1}^{s} u_{r} y_{r o}}{\sum_{i=1}^{m} v_{i} x_{i o}} \\
\text { s.t. } & \alpha \leq \frac{\sum_{r=1}^{s} u_{r} y_{r j}}{\sum_{i=1}^{m} v_{i} x_{i j}} \leq 1, \quad j=1, \ldots, n, \\
& u_{r}, v_{i} \geq \varepsilon, \quad r=1, \ldots, s ; i=1, \ldots, m .
\end{aligned}
$$

The introduced models as in (13) are called the bounded DEA schemes, which can be converted into LP models $[6,20]$. Consider $\theta_{o}^{U *}$ and $\theta_{o}^{L *}$ as the maximum and minimum values for the above-illustrated objective function, respectively; then, values construct an interval known as the efficiency interval. For $\mathrm{DMU}_{o}$, the upper-bound of this interval is calculated by considering the "optimistic" efficiency, whereas the lower-bound is calculated by considering the "pessimistic" efficiency. Therefore, this set of bounds is considered as the range of efficiency for $\mathrm{DMU}_{o}$, and it is represented by $\left[\theta_{o}^{L *}, \theta_{o}^{U *}\right]$.

The numerical example presented in Section 2 is used to compare between Azizi's and Entani et al.s methods. The input and output values of IDMU are determined by Azizi's method as follows:

$$
\begin{aligned}
x_{1}^{\min }=\min _{j}\left\{x_{1 j}\right\} & =1, \quad y_{1}^{\max }=\max _{j}\left\{y_{1 j}\right\}=7, \\
y_{2}^{\max } & =\max _{j}\left\{y_{2 j}\right\}=8 .
\end{aligned}
$$

The "pessimistic" efficiency of IDMU is equal to $\varphi_{\mathrm{IDMU}}^{*}=$ 2.8752. Finally, to calculate the efficiency interval of the DMUs, the bounded DEA schemes (13) are used to calculate $\alpha$ :

$$
\theta_{\min }^{*}=0.5217, \quad \varphi_{\mathrm{IDMU}}^{*}=2.8752 \Longrightarrow \alpha=\frac{0.5217}{2.8752}=0.1814 \text {. }
$$

The results of models (13) with respect to the efficiency interval of each DMU are shown in Table 2. Based on these results, the bounded DEA models (13) determine $\mathrm{DMU}_{\mathrm{A}}$, $\mathrm{DMU}_{\mathrm{E}}$, and $\mathrm{DMU}_{\mathrm{J}}$ as the "optimistic" efficient DMUs. The $\mathrm{DMU}_{\mathrm{A}}, \mathrm{DMU}_{\mathrm{B}}, \mathrm{DMU}_{\mathrm{F}}$, and $\mathrm{DMU}_{\mathrm{J}}$ are defined as the "pessimistic" inefficient DMUs. Finally, Azizi [6] used the A-index to compare and rank the interval efficiencies of these 10 DMUs. The results of this ranking are shown in the last column of Table 2. For example, $\mathrm{DMU}_{\mathrm{G}}$ is not a "DEA efficient" unit; it has better performance (rank 2) than both $\mathrm{DMU}_{\mathrm{A}}$ and $\mathrm{DMU}_{\mathrm{J}}$, whereas $\mathrm{DMU}_{\mathrm{A}}$, which is located on the efficient frontier, was ranked as the third unit.

3.3. Measuring the Efficiency Score with a Common Set of Weights. Jahanshahloo et al. [14] proposed a ranking method in which a positive ideal line is defined. A common set of weights is also determined for all DMUs, and a new efficiency score is proposed for ranking the efficient DMUs.

The positive ideal DMU is defined as "a virtual DMU with the minimized inputs of all of the DMUs as its input and the maximized outputs of all of the DMUs as its output." [14].

The positive ideal DMU is given as follows:

$$
\begin{aligned}
\overline{\mathrm{DMU}} & =(\bar{X}, \bar{Y}), \\
\bar{x}_{i} & =\min \left\{x_{i j} \mid j=1, \ldots, n\right\}, \quad(i=1, \ldots, m), \\
\bar{y}_{r} & =\max \left\{y_{r j} \mid j=1, \ldots, n\right\}, \quad(r=1, \ldots, s) .
\end{aligned}
$$

A positive ideal line is defined as "a straight line that passes through the origin and positive ideal DMU with slope 1.0.” [14].

In Figure 2, the vertical and horizontal axes are set to be the virtual output (the weighted sum of $s$ outputs) and the virtual input (the weighted sum of $m$ inputs), respectively, "ox" is a positive ideal line, and $\overline{\mathrm{DMU}}=\left(\sum_{i=1}^{m} \bar{x}_{i} v_{i}^{\prime}, \sum_{r=1}^{s} \bar{y}_{r} u_{r}^{\prime}\right)$ is a positive ideal DMU.

Consider $\mathrm{DMU}_{N}$ and $\mathrm{DMU}_{M}$ with the sets of weights $u_{r}^{\prime}(r=1, \ldots, s)$ and $v_{i}^{\prime}(i=1, \ldots, m)$, respectively. In Figure 2, the respective coordinates of points $M^{\prime}$ and $N^{\prime}$ under the positive ideal line are $\left(\sum_{i=1}^{m} x_{i M} v_{i}^{\prime}, \sum_{r=1}^{s} y_{r M} u_{r}^{\prime}\right)$ and $\left(\sum_{i=1}^{m} x_{i N} v_{i}^{\prime}, \sum_{r=1}^{s} y_{r N} u_{r}^{\prime}\right)$.

The parameters $\Delta_{M^{\prime}}^{I}$ and $\Delta_{M^{\prime}}^{O}$ illustrate the virtual gaps between points $M^{\prime}$ and $M^{\prime p}$ on the horizontal and vertical axis, respectively. In addition, the parameters $\Delta_{N^{\prime}}^{I}$ and $\Delta_{N^{\prime}}^{O}$ illustrate the gaps for points $N^{\prime}$ and $N^{\prime} p$. The total virtual gap to the positive ideal line is equal to $\Delta_{M^{\prime}}^{I}+\Delta_{M^{\prime}}^{O}+\Delta_{N^{\prime}}^{I}+\Delta_{N^{\prime}}^{O}$. An optimal set of weights $u_{r}^{*}(r=1, \ldots, s)$ and $v_{i}^{*}(i=1, \ldots, m)$ is determined based on [14]. These sets would be determined so that the points $M^{*}$ and $N^{*}$ under the positive ideal line 


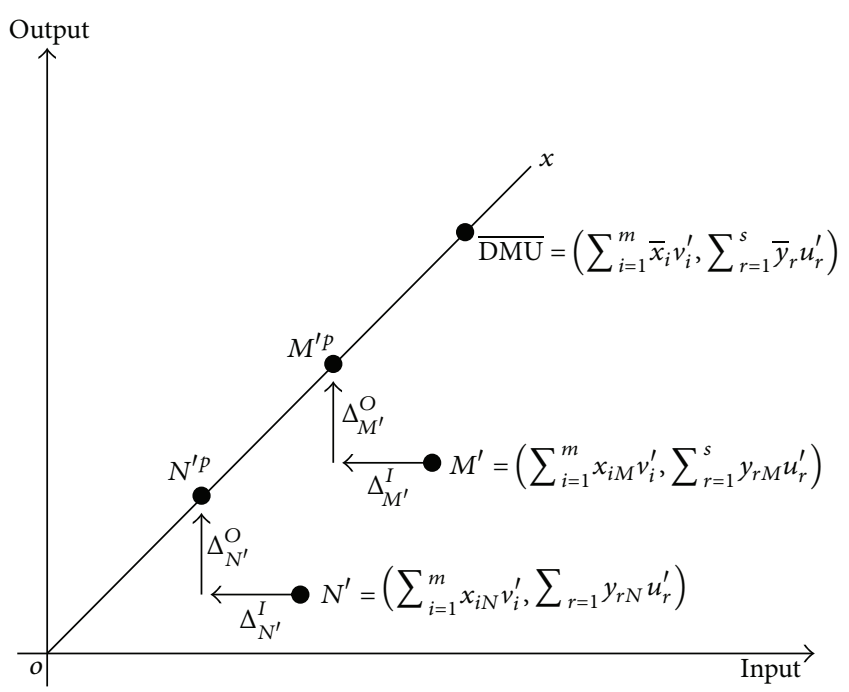

Figure 2: An analysis of the gap for the DMUs below the virtual positive ideal line.

could be as close as possible to their projection points $M^{* p}$ and $N^{* P}$ on the positive ideal line. That is, using the optimal weights, the total virtual distance $\Delta_{M^{*}}^{I}+\Delta_{M^{*}}^{O}+\Delta_{N^{*}}^{I}+\Delta_{N^{*}}^{O}$ to the positive ideal line is the shortest distance to both DMUs. The constraint is also a fraction in which the weighted sum of the outputs plus the vertical gap $\Delta_{j}^{O}$ is the numerator and the weighted sum of the inputs minus the horizontal virtual gap $\Delta_{j}^{I}$ is the denominator. Based on this constraint, a simultaneous upwards and leftwards direction is the closest orientation to the positive ideal line. However, the ratio of the numerator to the denominator equals 1.0, which implies that the projection point on the positive ideal line is achieved. Therefore, the proposed scheme is given as follows:

$$
\begin{array}{ll}
\Delta^{*}= & \min \sum_{j=1}^{n}\left(\Delta_{j}^{I}+\Delta_{j}^{O}\right) \\
\text { s.t. } & \frac{\sum_{r=1}^{s} u_{r} \bar{y}_{r}}{\sum_{i=1}^{m} v_{i} \bar{x}_{i}}=1, \\
& \frac{\sum_{r=1}^{s} u_{r} y_{r j}+\Delta_{j}^{O}}{\sum_{i=1}^{m} v_{i} x_{i j}-\Delta_{j}^{I}}=1, \\
& \Delta_{j}^{I}, \Delta_{j}^{O} \geq 0, \\
& u_{r} \geq \varepsilon>0, \quad r=1, \ldots, s, \\
& v_{i} \geq \varepsilon>0, \quad i=1, \ldots, m,
\end{array}
$$

where $\varepsilon$ is a positive Archimedean infinitesimal constant. Let $\Delta_{j}^{O}+\Delta_{j}^{I}$ be $\Delta_{j}$; then, (17) is simplified to the following linear programming:

$$
\begin{array}{ll}
\Delta^{*}= & \min \sum_{j=1}^{n} \Delta_{j} \\
\text { s.t. } \quad & \sum_{r=1}^{s} u_{r} \bar{y}_{r}-\sum_{i=1}^{m} v_{i} \bar{x}_{i}=0, \\
& \sum_{r=1}^{s} u_{r} y_{r j}-\sum_{i=1}^{m} v_{i} x_{i j}+\Delta_{j}=0, \\
& \Delta_{j} \geq 0, \quad \\
& u_{r} \geq \varepsilon>0, \quad r=1, \ldots, s, \\
& v_{i} \geq \varepsilon>0, \quad i=1, \ldots, m .
\end{array}
$$

When $\mathrm{DMU}_{j}$ is located on the positive ideal line, the definition of the common weight analysis efficiency (CWAefficiency) score for $\mathrm{DMU}_{j}$ is used. This definition was proposed by Liu and Peng [21] as

$$
\xi_{j}^{*}=\frac{\sum_{r=1}^{s} y_{r j} u_{r}^{*}}{\sum_{i=1}^{m} x_{i j} v_{i}^{*}} .
$$

Thus, $\mathrm{DMU}_{j}$ is determined to be CWA efficient when $\Delta_{j}^{*}=0$ or $\xi_{j}^{*}=1$; else, $\mathrm{DMU}_{j}$ is determined to be CWA inefficient. The performance of $\mathrm{DMU}_{j}$ is better than $\mathrm{DMU}_{i}$ if $\xi_{j}^{*}>\xi_{i}^{*}$.

In traditional DEA, the CCR model lets the DMUs calculate their maximum efficiency scores by the most favorable weights. This model has some deficits. For instance, when the efficiencies of different DMUs are measured by various sets of weights, the decision maker cannot compare and rank the DMUs on the same basis. For example, in Azizi's model [6], the most favorable $u$ and $v$ are obtained to maximize the efficiency score for each DMU. The importance rate for each output and input is different because of the choice of unique $u$ and $v$ for each DMU. In other words, this model measures the ratio of the weighted sum of the outputs to the weighted sum of the inputs. Therefore, a DMU being located in the production possibility set (PPS), which is an inefficient DMU, has a better rank with respect to an efficient DMU. As can be seen in Figure $1, D_{G}$ which is an inefficient DMU has a better rank with respect to efficient $\mathrm{DMU}_{\mathrm{A}}$.

\section{The Proposed Method}

In the proposed method, the efficiency score of $\mathrm{DMU}_{j}(j=$ $1, \ldots, n)$ is presented as an interval $\left[\xi_{j}^{* L}, \xi_{j}^{* U}\right]$ in which the $\xi_{j}^{* L}$ and $\xi_{j}^{* U}$ are the lower- and upper-bound of the efficiency interval for $\mathrm{DMU}_{j}$, respectively.

4.1. Calculating the Lower-Bound of the Efficiency Interval. To obtain the lower-bound, $\xi_{j}^{* L}$, of the efficiency interval for $\mathrm{DMU}_{j}$, which is in $(0,1],(19)$ is used in which $u_{r}^{*}$ and $v_{i}^{*}$ are determined based on model (18). When the positive ideal DMU is among the real DMUs, the value of the lower-bound is equal to 1 . 


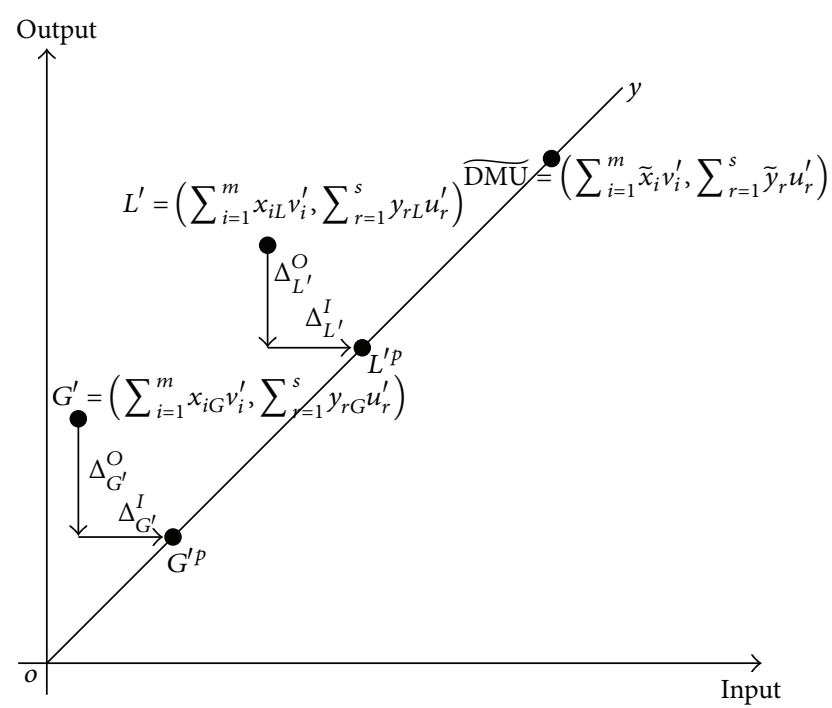

FIgURE 3: An analysis of the gap for the DMUs above the virtual anti-ideal line.

4.2. Calculating the Upper-Bound of the Efficiency Interval. To achieve the upper-bound of the efficiency interval for $\mathrm{DMU}_{j}$, $\xi_{j}^{* U}$, which is in $[1,+\infty)$, the following definitions are defined.

Definition 1. An anti-ideal DMU is a virtual DMU that has the maximum inputs of all of DMUs as its input and the minimum outputs of all of DMUs as its output.

This DMU is given as follows:

$$
\begin{aligned}
\widetilde{\mathrm{DMU}} & =(\widetilde{X}, \widetilde{Y}), \\
\widetilde{x}_{i} & =\max \left\{x_{i j} \mid j=1, \ldots, n\right\}, \quad(i=1, \ldots, m), \\
\widetilde{y}_{r} & =\min \left\{y_{r j} \mid j=1, \ldots, n\right\}, \quad(r=1, \ldots, s) .
\end{aligned}
$$

Definition 2. An anti-ideal line is a direct line that passes through the origin and the anti-ideal DMU with slope 1.0.

In Figure 3, the vertical axis is set to be the virtual output, which is the weighted sum of $s$ outputs, and the horizontal axis is set to be the virtual input, which is the weighted sum of $m$ inputs. In Figure 3, "oy" illustrates an anti-ideal line and $\widetilde{\mathrm{DMU}}=\left(\sum_{i=1}^{m} \tilde{x}_{i} v_{i}^{\prime}, \sum_{r=1}^{s} \tilde{y}_{r} u_{r}^{\prime}\right)$ denotes an anti-ideal DMU.

Therefore, the coordinate of the point $L^{\prime}$ above the antiideal line is $\left(\sum_{i=1}^{m} x_{i L} v_{i}^{\prime}, \sum_{r=1}^{s} y_{r L} u_{r}^{\prime}\right)$ and the coordinate of the point $G^{\prime}$ above the anti-ideal line is $\left(\sum_{i=1}^{m} x_{i G} v_{i}^{\prime}, \sum_{r=1}^{s} y_{r G} u_{r}^{\prime}\right)$.

Whereas the virtual gap between the points $L^{\prime}$ and $L^{\prime p}$ on the horizontal axis is denoted by $\Delta_{L^{\prime}}^{I}$, the virtual gap between the points $L^{\prime}$ and $L^{\prime p}$ on the vertical axis is denoted by $\Delta_{L^{\prime}}^{O}$. Similarly, for the points $G^{\prime}$ and $G^{\prime p}$, the gaps are denoted by $\Delta_{G^{\prime}}^{I}$ and $\Delta_{G^{\prime}}^{O}$, respectively. The total virtual distance from the anti-ideal line is $\Delta_{G^{\prime}}^{I}+\Delta_{G^{\prime}}^{O}+\Delta_{L^{\prime}}^{I}+\Delta_{L^{\prime}}^{O}$. An optimum set of weights $u_{r}^{*}(r=1, \ldots, s)$ and $v_{i}^{*}(i=1, \ldots, m)$ are determined so that both of the points $G^{*}$ and $L^{*}$ above the anti-ideal line are as far as possible from their projection points $G^{* p}$ and $L^{* p}$ on the anti-ideal line.
TABLE 3: The interval efficiencies of the proposed method for 10 DMUs and their ranks.

\begin{tabular}{lcc}
\hline DMU & $\begin{array}{c}\text { Interval efficiency } \\
\text { (proposed model) }\end{array}$ & $\begin{array}{c}\text { Rank } \\
\text { (proposed model) }\end{array}$ \\
\hline $\mathrm{A}$ & {$[0.6000,7.9986]$} & 2 \\
$\mathrm{~B}$ & {$[0.3333,2.9998]$} & 10 \\
$\mathrm{C}$ & {$[0.5333,5.9992]$} & 4 \\
$\mathrm{D}$ & {$[0.4000,3.0000]$} & 8 \\
$\mathrm{E}$ & {$[0.6667,6.9992]$} & 1 \\
$\mathrm{~F}$ & {$[0.4000,2.0004]$} & 9 \\
$\mathrm{G}$ & {$[0.6000,4.9998]$} & 3 \\
$\mathrm{H}$ & {$[0.4667,2.0006]$} & 7 \\
$\mathrm{I}$ & {$[0.5333,2.0008]$} & 5 \\
$\mathrm{~J}$ & {$[0.5333,1.0012]$} & 6 \\
\hline
\end{tabular}

In other words, by adopting optimal weights, the sum of the virtual gaps is $\Delta_{L^{*}}^{I}+\Delta_{L^{*}}^{O}+\Delta_{G^{*}}^{I}+\Delta_{G^{*}}^{O}$ which is the longest relative to both DMUs. To achieve this goal, the following model is presented:

$$
\begin{array}{ll}
\Delta^{*}= & \max \sum_{j=1}^{n} \Delta_{j} \\
\text { s.t. } & \sum_{r=1}^{s} u_{r} \tilde{y}_{r}-\sum_{i=1}^{m} v_{i} \tilde{x}_{i}=0, \\
& \sum_{r=1}^{s} u_{r} y_{r j}-\sum_{i=1}^{m} v_{i} x_{i j}-\Delta_{j}=0, \\
& \sum_{i=1}^{m} v_{i}+\sum_{r=1}^{s} u_{r}=1, \quad(*) \\
& \Delta{ }_{j} \geq 0, \quad r=1, \ldots, s, \\
& u_{r} \geq \varepsilon>0, \quad r=1, \ldots, m . \\
& v_{i} \geq \varepsilon>0, \quad i=
\end{array}
$$

In some cases, the optimal solution of (21) could be unbounded. To avoid this situation, the constraint $\sum_{i=1}^{m} v_{i}+$ $\sum_{r=1}^{s} u_{r}=1$ is added to the other constraints in model (21). Similarly, to obtain the upper-bound of the interval efficiency of $\mathrm{DMU}_{j}, \xi_{j}^{* U},(19)$ is used, where $u_{r}^{*}$ and $v_{i}^{*}$ are determined based on (21). When the anti-ideal DMU is among the real DMUs, the value of the upper-bound is equal to 1 . Providing an initial basic feasible solution (IBFS) for the above proposed DEA models can practically decrease fifty percent of the whole computations. Interested readers can refer to the paper by Toloo et al. [22] for more details of finding an IBFS for these proposed models.

The proposed model was run to obtain the interval efficiency of the 10 DMUs used by Entani et al. [2] and Azizi [6]. The results are shown in Table 3 . In the next section, the proposed method is compared with other previous methods based on this interval efficiency. The DMUs will be ranked using this interval efficiency. In Table 3, the DMUs that have 
the higher lower-bound have a better rank. If the lowerbound of two DMUs is equal, then the DMU with the higher upper-bound will have a better rank.

\section{Results and Discussion}

The proposed interval efficiency is used to rank DMUs. The DMU with the highest lower-bound has the best rank. If two DMUs have the same lower-bound, then the DMU with the higher upper-bound will have the better rank. It is logical that the worst DMU is a DMU that is located on the worst point of the inefficient frontier. The DMUs that are located between the "pessimistic" and "optimistic" frontiers have middle rank. All of the DMUs that are located near the "optimistic" frontier have a better rank, while all of the DMUs that are located near the "pessimistic" frontier have a worse rank.

As it can be seen in Figure 1, $\mathrm{DMU}_{\mathrm{E}}$ is located on two strong efficient hyperplanes. Based on the proposed model, $\mathrm{DMU}_{\mathrm{E}}$ has the highest lower-bound and it is ranked as the best DMU.

In our proposed method, $\mathrm{DMU}_{\mathrm{A}}$ and $\mathrm{DMU}_{\mathrm{G}}$ have the same lower-bound. The $\mathrm{DMU}_{\mathrm{A}}$ has the higher upper-bound with respect to $\mathrm{DMU}_{\mathrm{G}}$. Therefore, $\mathrm{DMU}_{\mathrm{A}}$ has a better rank position. In Azizi's ranking, $\mathrm{DMU}_{\mathrm{G}}$ is in rank position 2, but this DMU is dominated by other DMUs and is not even located on the efficient frontier, while $\mathrm{DMU}_{\mathrm{A}}$ that is located on one strong efficient hyperplane is in rank position 3. In Azizi's ranking, $\mathrm{DMU}_{\mathrm{G}}$ was ranked better with respect to $\mathrm{DMU}_{\mathrm{A}}$, whereas, in the proposed model, $\mathrm{DMU}$ A has a better rank with respect to $\mathrm{DMU}_{\mathrm{G}}$, that is, more realistic.

As it can be seen in Figure 1, DMU $\mathrm{F}$ is located on the inefficient frontier and $\mathrm{DMU}_{\mathrm{D}}$ is located between the efficient and inefficient frontiers. In Azizi [6], $\mathrm{DMU}_{\mathrm{F}}$ has a better rank with respect to $\mathrm{DMU}_{\mathrm{D}}$, while in the proposed method $\mathrm{DMU}_{\mathrm{F}}$ has a worse rank with respect to $\mathrm{DMU}_{\mathrm{D}}$, that is, more acceptable.

In both Azizi's model and the proposed model, $\mathrm{DMU}_{\mathrm{E}}$ has the best rank. $\mathrm{DMU}_{\mathrm{E}}$ is located on the two strong efficient hyperplanes which implies the best efficiency for $\mathrm{DMU}_{\mathrm{E}}$. The $\mathrm{DMU}_{\mathrm{B}}$ is in rank position 10, which is the worst rank among all of the DMUs. The $\mathrm{DMU}_{\mathrm{B}}$ is located on the two weak inefficient hyperplanes which implies the worst inefficiency for $\mathrm{DMU}_{\mathrm{B}}$.

In the proposed method, $\mathrm{DMU}_{\mathrm{D}}$ and $\mathrm{DMU}_{\mathrm{H}}$ are in rank positions 8 and 7 , respectively. These DMUs are close to the pessimistic frontier, but they are located in better positions with respect to $\mathrm{DMU}_{\mathrm{B}}$ and $\mathrm{DMU}_{\mathrm{F}}$, which are located on the inefficient frontier. Therefore, the DMUs that are located between the "pessimistic" and "optimistic" frontiers are ranked correctly.

\section{Conclusion and Future Work}

In this paper, both the "optimistic" and "pessimistic" relative efficiencies are used simultaneously. The overall performance of the DMUs is measured whereby an integration of both the best and the worst relative efficiencies is in the form of an interval. The lower- and upper-bound of the interval efficiency are achieved by employing a common set of weights that is determined by the positive ideal and anti-ideal lines. Then, the DMUs were ranked using this interval. In this method, all of the DMUs are compared simultaneously with this common set of weights. The capabilities of the proposed DEA method are illustrated by a numerical example. The proposed method measures the efficiency and ranks all of the efficient and inefficient DMUs, simultaneously. Therefore, the proposed interval efficiency method is simpler than the other methods and it is convenient to use in comparison with Entani et al. and Azizi's interval models. The proposed method is also reliable, such that it presents a more acceptable ranking procedure that leads to logical and acceptable results in comparison with previous works. Computing the appropriate value of $\varepsilon$ for the proposed models can be considered as a future direction of this study.

\section{Conflict of Interests}

The authors declare that there is no conflict of interests regarding the publication of this paper.

\section{Acknowledgments}

This research is supported by a Research University Grant (00K55) awarded by Universiti Teknologi Malaysia (UTM). The researchers are thankful for this financial support. The authors also are thankful to the anonymous referees for many helpful suggestions for improving the presentation of this paper.

\section{References}

[1] A. Charnes, W. W. Cooper, and E. Rhodes, "Measuring the efficiency of decision making units," European Journal of Operational Research, vol. 2, no. 6, pp. 429-444, 1978.

[2] T. Entani, Y. Maeda, and H. Tanaka, "Dual models of interval DEA and its extension to interval data," European Journal of Operational Research, vol. 136, no. 1, pp. 32-45, 2002.

[3] Y.-M. Wang and Y. Luo, "DEA efficiency assessment using ideal and anti-ideal decision making units," Applied Mathematics and Computation, vol. 173, no. 2, pp. 902-915, 2006.

[4] Y.-M. Wang and J.-B. Yang, "Measuring the performances of decision-making units using interval efficiencies," Journal of Computational and Applied Mathematics, vol. 198, no. 1, pp. 253267, 2007.

[5] G. R. Jahanshahloo, F. Hosseinzadeh Lotfi, V. Rezaie, and M. Khanmohammadi, "Ranking DMUs by ideal points with interval data in DEA," Applied Mathematical Modelling, vol. 35, no. 1, pp. 218-229, 2011.

[6] H. Azizi, "The interval efficiency based on the optimistic and pessimistic points of view," Applied Mathematical Modelling, vol. 35, no. 5, pp. 2384-2393, 2011.

[7] A. Sengupta and T. K. Pal, "On comparing interval numbers," European Journal of Operational Research, vol. 127, no. 1, pp. 2843, 2000.

[8] Y.-M. Wang, Y. Luo, and Y.-X. Lan, "Common weights for fully ranking decision making units by regression analysis," Expert Systems with Applications, vol. 38, no. 8, pp. 9122-9128, 2011.

[9] F. Hosseinzadeh Lotfi, G. R. Jahanshahloo, M. Khodabakhshi, M. Rostamy-Malkhlifeh, Z. Moghaddas, and M. Vaez-Ghasemi, 
"A review of ranking models in data envelopment analysis," Journal of Applied Mathematics, vol. 2013, Article ID 492421, 20 pages, 2013.

[10] J. A. Ganley and S. A. Cubbin, Public Sector Efficiency Measurement: Applications of Data Envelopment Analysis, Elsevier Science, Amsterdam, The Netherlands, 1992.

[11] Y. Roll, W. D. Cook, and B. Golany, "Controlling factor weights in data envelopment analysis," IIE Transactions, vol. 23, no. 1, pp. 2-9, 1991.

[12] Y. Roll and B. Golany, "Alternate methods of treating factor weights in DEA," Omega, vol. 21, no. 1, pp. 99-109, 1993.

[13] Z. Sinuany-Stern and L. Friedman, "DEA and the discriminant analysis of ratios for ranking units," European Journal of Operational Research, vol. 111, no. 3, pp. 470-478, 1998.

[14] G. R. Jahanshahloo, F. Hosseinzadeh Lotfi, M. Khanmohammadi, M. Kazemimanesh, and V. Rezaie, "Ranking of units by positive ideal DMU with common weights," Expert Systems with Applications, vol. 37, no. 12, pp. 7483-7488, 2010.

[15] P. Andersen and N. C. Petersen, "A procedure for ranking efficient units in data envelopment analysis," Management Science, vol. 39, no. 10, pp. 1261-1264, 1993.

[16] W. D. Cook, M. Kress, and L. M. Seiford, "Prioritization models for frontier decision making units in DEA," European Journal of Operational Research, vol. 59, no. 2, pp. 319-323, 1992.

[17] G. R. Jahanshahloo, H. V. Junior, F. Hosseinzadeh Lotfi, and D. Akbarian, "A new DEA ranking system based on changing the reference set," European Journal of Operational Research, vol. 181, no. 1, pp. 331-337, 2007.

[18] G. R. Amin and M. Toloo, "A polynomial-time algorithm for finding $\epsilon$ in DEA models," Computers \& Operations Research, vol. 31, no. 5, pp. 803-805, 2004.

[19] C. Parkan and Y. Wang, "The worst possible relative efficiency analysis based on inefficient production frontier," Working Paper, Department of Management Sciences, City University of Hong Kong, 2000.

[20] A. Charnes and W. W. Cooper, "Programming with linear fractional functionals," Naval Research Logistics Quarterly, vol. 9, no. 3-4, pp. 181-186, 1962.

[21] F.-H. F. Liu and H. H. Peng, "Ranking of units on the DEA frontier with common weights," Computers \& Operations Research, vol. 35, no. 5, pp. 1624-1637, 2008.

[22] M. Toloo, A. Masoumzadeh, and M. Barat, "Finding an initial basic feasible solution for DEA models with an application on bank industry," Computational Economics, 2014. 


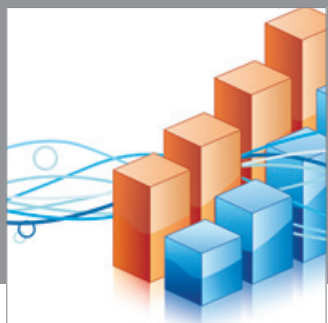

Advances in

Operations Research

mansans

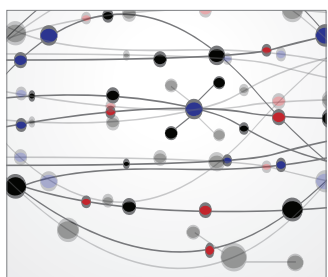

The Scientific World Journal

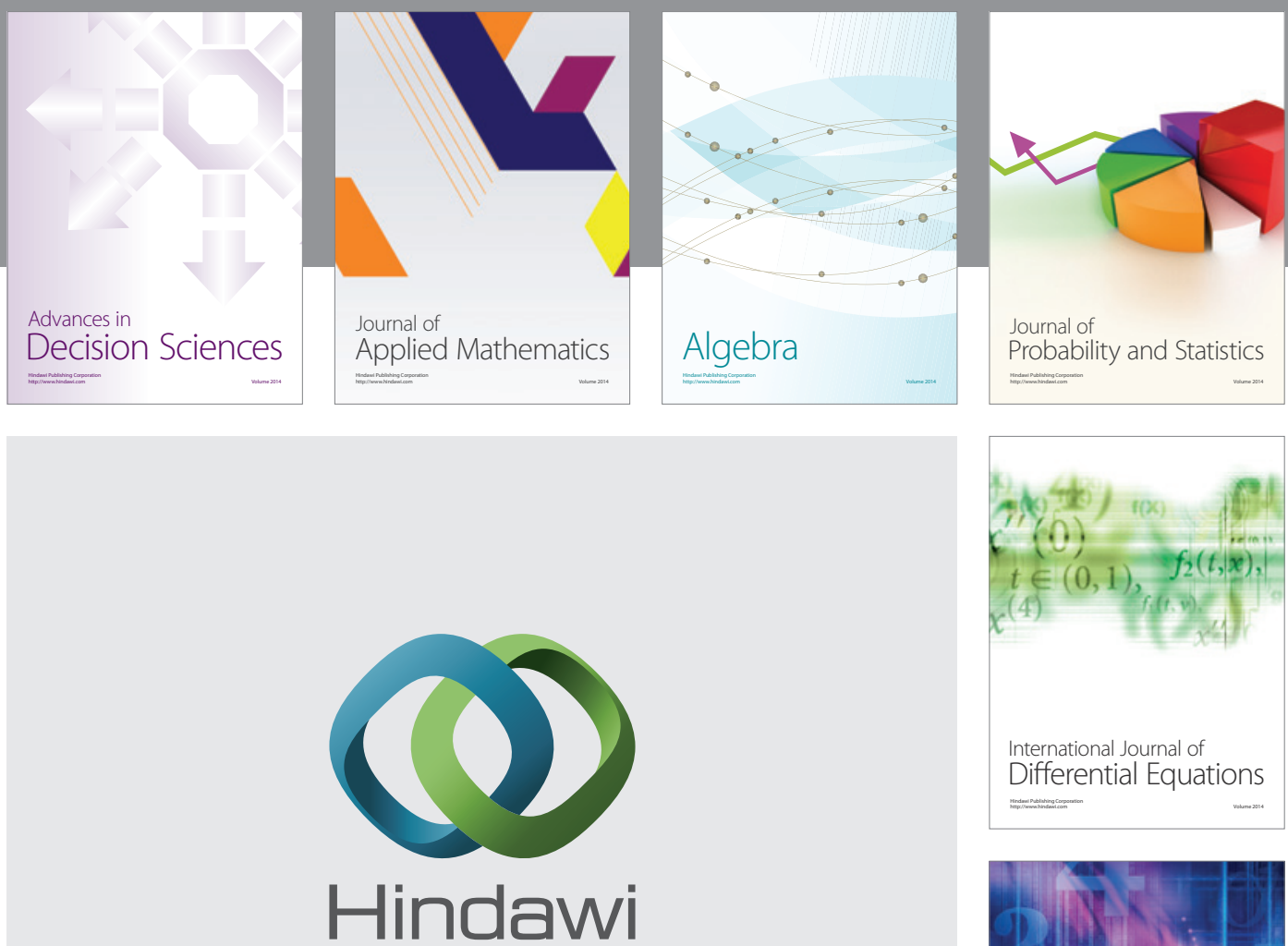

Submit your manuscripts at http://www.hindawi.com
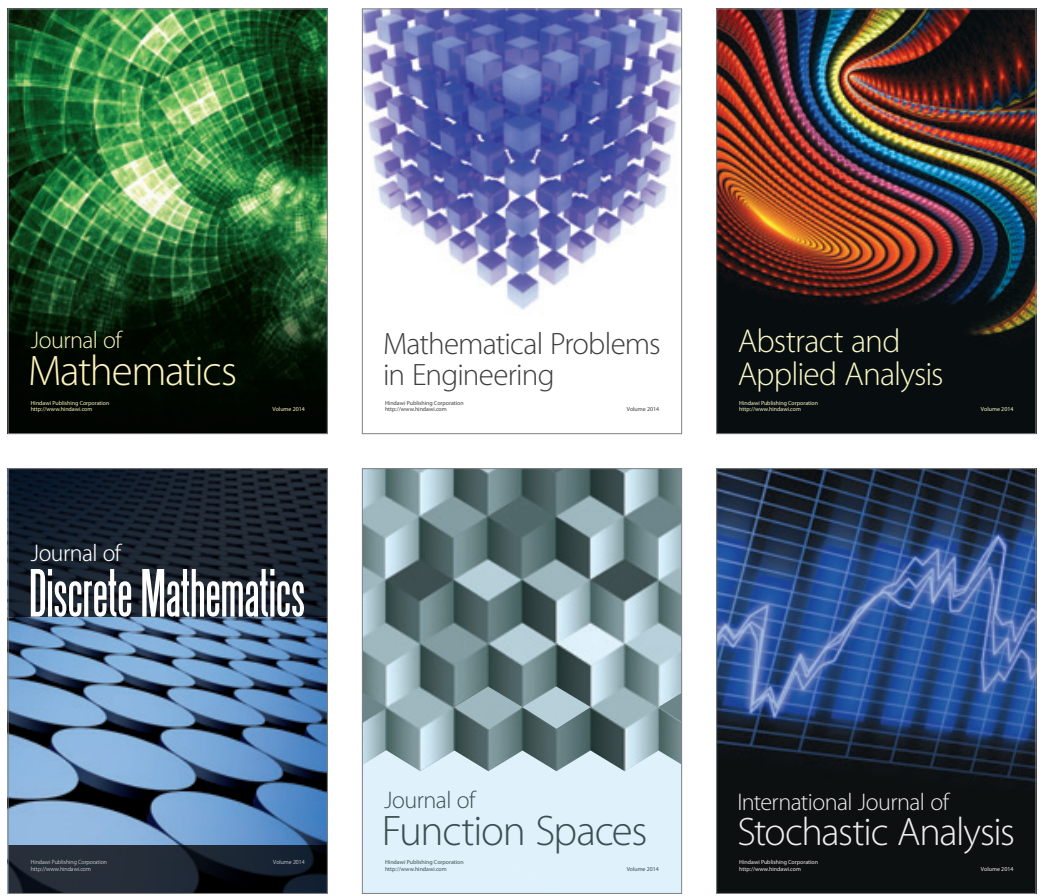

Journal of

Function Spaces

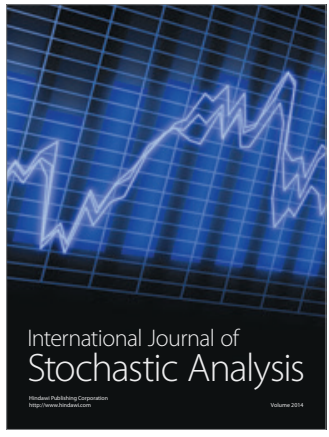

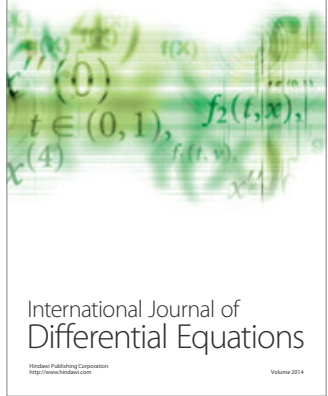
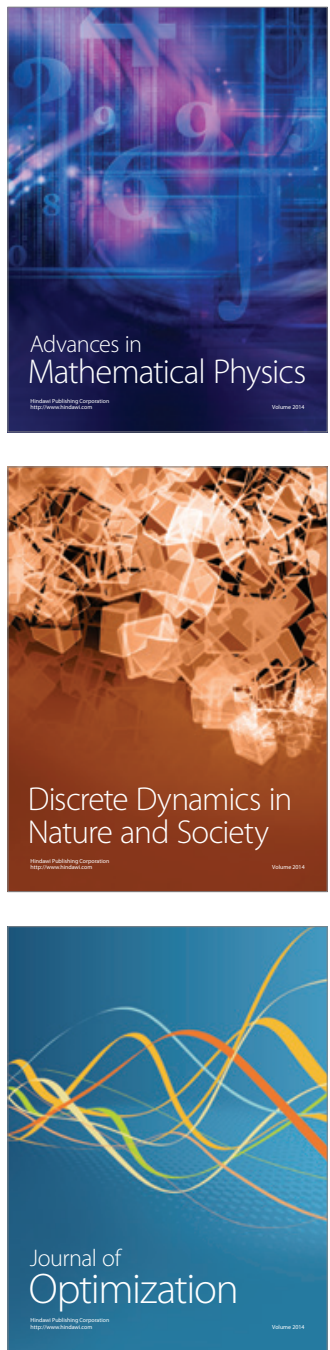